\title{
Cross-Current SOI MOSFET and Application to Multiple Voltage Reference Circuits
}

\author{
Yasuhisa Omura $^{1}$, Yoshio Mimura ${ }^{1}$, and Masakazu Kitagawa ${ }^{2}$ \\ ${ }^{1}$ ORDIST, Grad. School of Sci. and Eng., Kansai University \\ 3-3-35, Yamate-cho, Suita, Osaka 564-8680, Japan \\ Phone: +81-6-6368 -1121 E-mail: omuray@ipcku.kansai-u.ac.jp \\ ${ }^{2}$ Faculty of Eng., Kansai University \\ 3-3-35, Yamate-cho, Suita, Osaka 564-8680, Japan
}

\section{Introduction}

One of the authors (Omura) proposed the cross-current tetrode SOI MOSFET (XCT-SOI MOSFET) and examined analog applications [1]. Though the scaling feasibility of XCT-like devices has been studied recently [2], we think $\mathrm{XCT}$ devices will yield new applications such as high-voltage devices and SRAM memory cells with high noise margin. In order to assess those applications in sufficient detail, device models are needed to perform circuit simulations. Since Y. Azuma et al. has recently proposed an advanced device model for the XCT MOSFET [3, 4], we are now able to study device applications.

This paper proposes switched multiple-reference-voltage supplier circuits for low-power SOI LSIs. We examine their functions by circuit simulations (SPICE) on the basis of measured XCT MOSFET parameters. The usefulness of the XCT SOI MOSFET is demonstrated.

\section{Device Structure and Assumptions for Simulations}

Schematic device structure is already shown in [1]. In an XCT device, the n-channel MOSFET and p-channel JFET are self-merged and the electron current of the nMOSFET is relayed to the hole current of pJFET in series. The XCT device offers negative differential conductance in the saturation region of drain current [1,3]; device parameters of fabricated devices are summarized in Table 1 . Since the XCT device has active body contact, from pJFET, the bodyfloating effect is eliminated automatically.

Table. 1. Physical parameters of fabricated devices.

\begin{tabular}{lcc}
\hline Device parameters & Values & {$[\mathrm{units}]$} \\
\hline Nominal body doping, $N_{A}$ & $1 \times 10^{16}$ & {$\left[\mathrm{~cm}^{-3}\right]$} \\
Gate width, $W n / W p$ & $10 / 20$ & {$[\mu \mathrm{m}]$} \\
Gate length, $L$ & 2 & {$[\mu \mathrm{m}]$} \\
Gate oxide thickness, $t_{O x}$ & 30 & {$[\mathrm{~nm}]$} \\
SOI layer thickness, $t_{S O I}$ & 350 & {$[\mathrm{~nm}]$} \\
\hline
\end{tabular}

In circuit simulations, we assume the equivalent circuit for nXCT device as shown in Fig. 1(a). We examined that the equivalent circuit reproduced the $\mathrm{FV}$ characteristics of
XCT device. We determined the SPICE simulation parameters using the device parameters shown in Table 1.

\section{Proposal of Voltage Reference Circuits and simulation Results}

We first propose a two reference voltage circuits, each with one XCT CMOS device, in Fig. 1(b) the XCT devices are shown as equivalent circuits. This is a simple CMOS configuration except for the independent gate terminals of $\mathrm{nXCT}$ device and pXCT device. It is assumed $V_{D D}=5 \mathrm{~V}$ and the gate voltage of pXCT device $\left(V_{G P}\right)$ basically holds a constant value; threshold voltages are $+0.6 \mathrm{~V}$ for $\mathrm{nXCT}$ and $0.6 \mathrm{~V}$ for $\mathrm{pXCT}$.

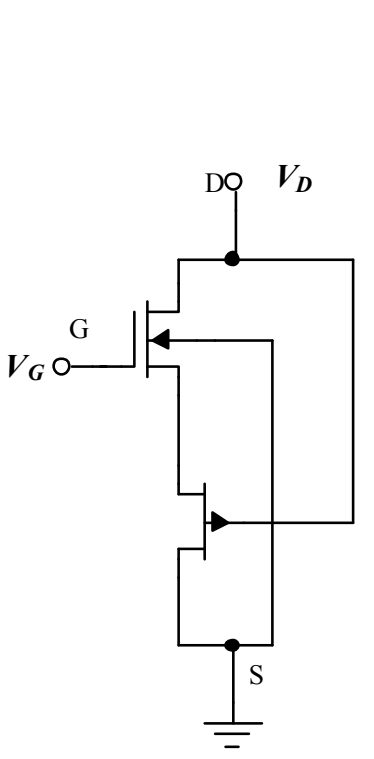

(a)

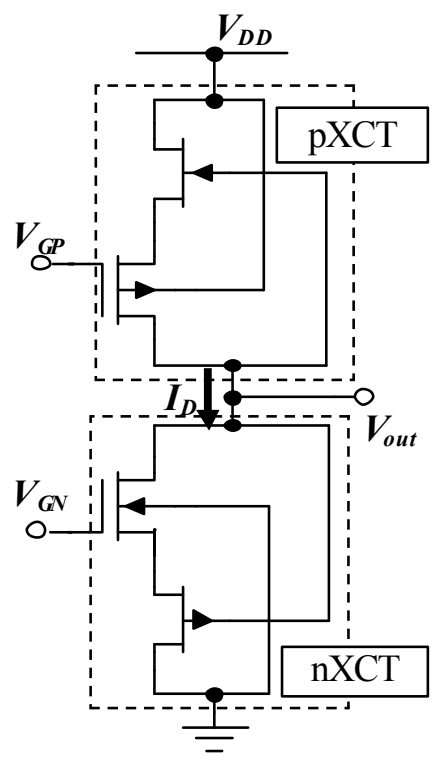

(b)
Fig. 1. Equivalent circuit model for nXCT device and XCT CMOS device.

Concept of switching the reference voltage is shown in Fig. 2, where simulated time sequences of the gate voltage of nXCT device $\left(V_{G N}\right)$, that of pXCT device $\left(V_{G P}\right)$, and output voltage $\left(V_{\text {out }} ; \quad V_{L} \leftarrow \rightarrow V_{H}\right)$. Switching mechanisms are schematically shown in Fig. 3, where the four figures 
correspond to notations '(a)' to '(d)' in the time evolution shown in Fig. 2.

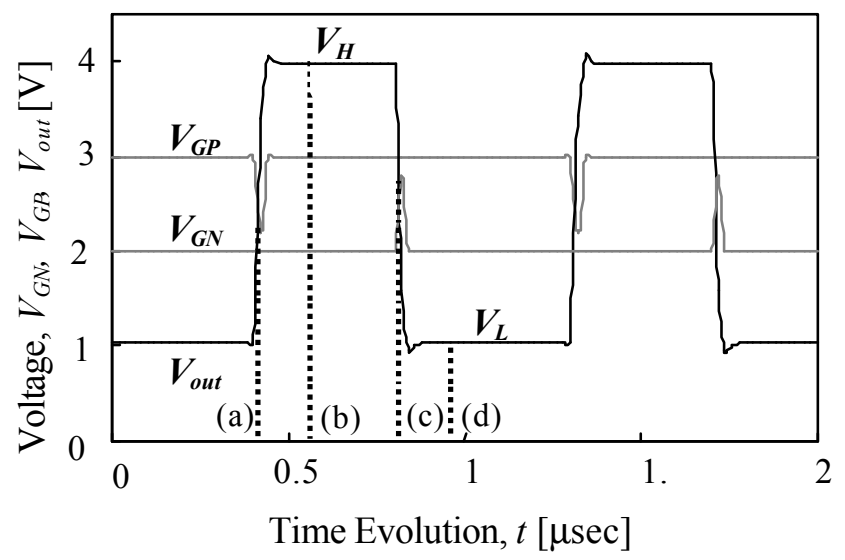

Fig. 2. Time evolution of $V_{G N}, V_{G P}$ and $V_{\text {out }}$.

In the operation shown in Fig. 2, a falling trigger is applied to $V_{G P}$ and a rising trigger is applied to $V_{G N}$. At the initial stage, $V_{D D}$ of $5 \mathrm{~V}$ is supplied to the circuit at first, whereupon $V_{G N}$ rises to $2 \mathrm{~V}$, and finally $V_{G P}$ rises to $3 \mathrm{~V}$; so, $V_{\text {out }}$ holds $V_{L}$ level at the initial stage. In Fig. 2, at $t=0.4$ $\mu$ sec, $V_{G P}$ steps down by $0.8 \mathrm{~V}$ for a short time and then rebounds, followed by a high $V_{\text {out }}$ level $\left(V_{H}\right)$; this process is illustrated in Figs. 3(a) and 3(b). The locus of the cross point of current curves of $\mathrm{nXCT}$ and $\mathrm{pXCT}$ devices moves from $V_{L}$ to a certain high level following the sequence shown in 3(a), and it finally t returns to $V_{H}$ when $V_{G P}$ returns to $3 \mathrm{~V}$ as shown in Fig. 3(b). At the next stage, $V_{G N}$ rises by $0.8 \mathrm{~V}$ at $\mathrm{t}=0.8 \mu \mathrm{sec}$ and returns to $2 \mathrm{~V}$; this trigger forces $V_{\text {out }}$ to step down to $V_{L}$. This sequence is illustrated in Figs. 3(c) and $3(d)$.

We also propose a three-reference-voltage circuit for practical convenience (not shown here). Basics of the operation mechanism are the same as those illustrated in Fig. 3.

\section{Acknowledgment}

The authors express their thanks to Drs. Hirobumi Watanabe and Hidenori Kato, Ricoh Corp., Japan for their technical cooperation in device fabrication.

\section{References}

[1] Y. Omura and K. Izumi, "High-gain Cross-current Tetrode MOSFET/SIMOX and Its Application", in Ext. Abstr., $18^{\text {th }}$ Int. Conf. Solid State Devices and Mat. (Tokyo, 1986) p. 715.

[2] B. J. Blalock, S. Cristoloveanu, B. M. Dufrene, F. Allibert, and M. Mojarradi, "The Multiple-Gate MOSFET-JFET Transistor”, Int. J. High Speed Electron. Syst., vol. 12, pp. 519-520, 2002.
[3] Y. Azuma, Y. Yoshioka, and Y. Omura, "Proposal of Cross-Current SOI MOSFET Model and Demonstration of CMOS Operations," Proc. 26 ${ }^{\text {th }}$ Electronic Material Symp. (EMS26) (Shiga, Biwako, July, 2007) pp. 25-28.

[4] Y. Azuma, Y. Yoshioka, and Y. Omura, "Cross-Current SOI MOSFET Model and Important Aspects of CMOS Operations," Ext. Abstr. Int. Conf. Solid State Devices and Mat. (Tsukuba, 2007) pp. 460-461.
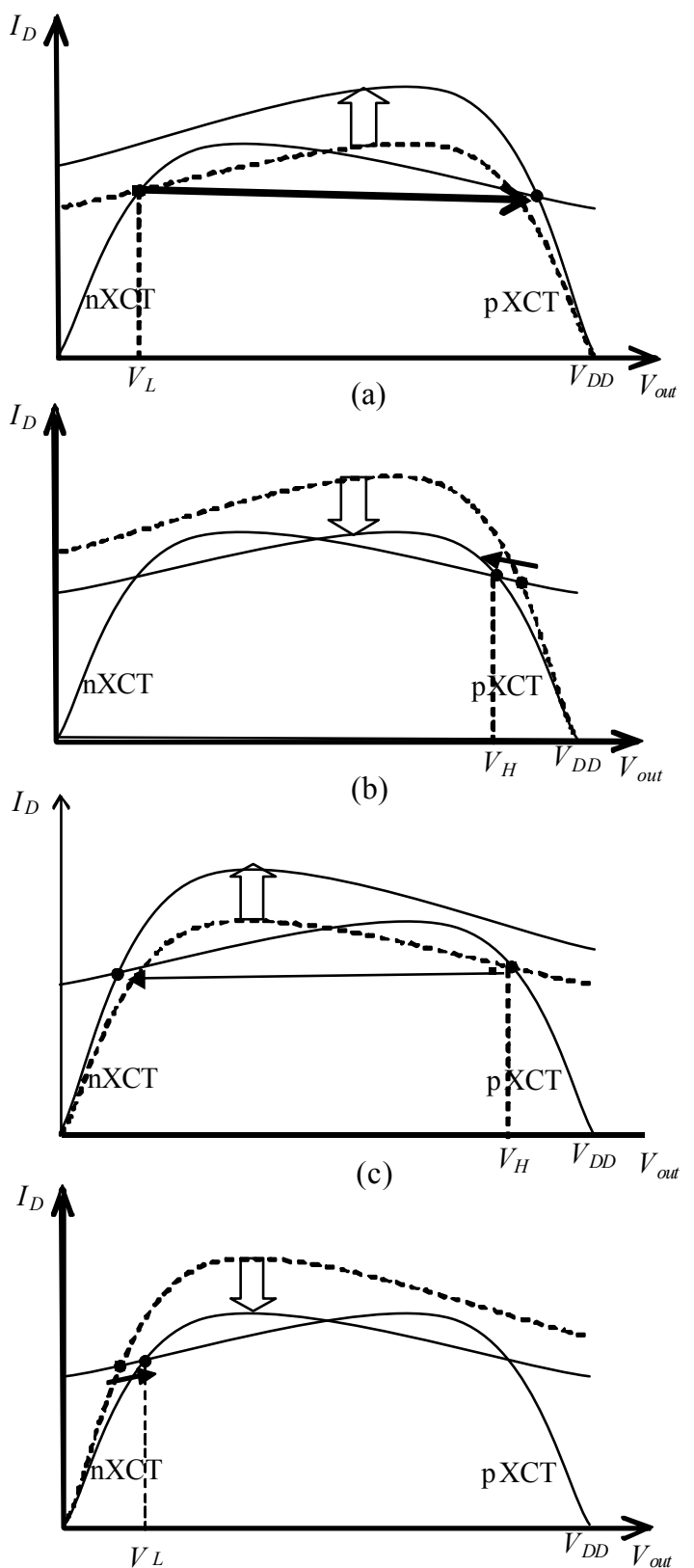

(d)

Fig. 3. Switching mechanism of reference voltages. 